\title{
General practitioners' perceptions of effective health care
}

\author{
Zelda Tomlin, Charlotte Humphrey, Stephen Rogers
}

Department of
Primary Care and
Population
Sciences, Royal Free
and University
College Medical
School, University
College London,
London NW3 2PF
Zelda Tomlin,
research fellow
Charlotte
Humphrey,
senior lecturer in
sociology
Stephen Rogers,
senior lecturer in
primary health care
Correspondence to:
Dr Humphrey
charlot@rfhom.
ac.uk

BMJ 1999;318:1532-5

\begin{abstract}
Objectives To explore general practitioners' perceptions of effective health care and its application in their own practice; to examine how these perceptions relate to assumptions about clinicians' values and behaviour implicit in the evidence based medicine approach.

Design A qualitative study using semistructured interviews.

Setting Eight general practices in North Thames region that were part of the Medical Research Council General Practice Research Framework. Participants 24 general practitioners, three from each practice.

Main outcome measures Respondents' definitions of effective health care, reasons for not practising effectively according to their own criteria, sources of information used to answer clinical questions about patients, reasons for making changes in clinical practice.

Results Three categories of definitions emerged: clinical, patient related, and resource related. Patient factors were the main reason given for not practising effectively; others were lack of time, doctors' lack of knowledge and skills, lack of resources, and "human failings." Main sources of information used in situations of clinical uncertainty were general practitioner partners and hospital doctors. Contact with hospital doctors and observation of hospital practice were just as likely as information from medical and scientific literature to bring about changes in clinical practice.

Conclusions The findings suggest that the central assumptions of the evidence based medicine paradigm may not be shared by many general practitioners, making its application in general practice problematic. The promotion of effective care in general practice requires a broader vision and a more pragmatic approach which takes account of practitioners' concerns and is compatible with the complex nature of their work.
\end{abstract}

\section{Introduction}

The concept of effectiveness has come to dominate the healthcare debate. The emergence of evidence of variations in practice, with accompanying doubts on the clinical effectiveness of some of those practices, ${ }^{12}$ has shown the need for a fundamental questioning of the way in which clinical decisions are made, identifying the reasons for such variation, and finding ways of addressing inappropriate variations. ${ }^{3}{ }^{4}$

Awareness of the latest scientific evidence and the ability to critically appraise and assess the applicability of this evidence have been identified as crucial ingredients that are missing in everyday medical practice, and evidence based medicine has emerged as a new paradigm. ${ }^{5}$ Evidence based medicine is defined as the "conscientious, explicit and judicious use of current best evidence in making decisions about the care of individual patients." ${ }^{6}$ This approach is underpinned by the assumption that practitioners regard clinical effectiveness as a priority and will be keen to act in a way that optimises this - that is, to question their clinical practice systematically and, where shortcomings are identified, change that practice in line with scientifically valid evidence.

Our research, carried out in general practice, explores the extent to which this emphasis on clinical effectiveness, self analysis, and information seeking is congruent with the modes of thinking and behaviour of a service general practitioner. We asked doctors to describe how they defined effectiveness in health care, whether they thought they always practised effectively and, if not, why not. We also asked them how they sought answers to clinical questions about individual patients and about recent changes in their own clinical practice and how these had come about.

\section{Participants and methods}

The study was carried out in the summer of 1997 at eight practices in the North Thames region that were members of the Medical Research Council General Practice Research Framework, a network of about 900 practices that have expressed a willingness to participate in MRC research projects. ${ }^{7}$ Framework practices are reimbursed for the time that practice nurses spend on the projects, have lockable filing facilities, and out of hours surgeries for study patients. The practices were recruited from those that responded to an invitation to participate in a feasibility study for a randomised controlled trial of implementation strategies to promote use of evidence based guidelines.

The data presented in this paper were collected as part of baseline interviews for the feasibility study, which were undertaken with 24 doctors, three from each practice. For each practice, those interviewed included at least one general practitioner with close links with the MRC, one woman general practitioner, and one general practitioner who was sceptical about joining the study, as identified during preliminary meetings. The interviews were semistructured and lasted about an hour. As well as the issues reported here, the interview covered the general practitioners' views on implementing research findings in clinical practice, practice guidelines and quality in health care, the characteristics of their practices, and their expectations of the feasibility study.

All the interviews were undertaken by the same researcher and were audio taped and subsequently transcribed. The transcripts were read to identify themes from individual responses, and these were then grouped into categories to produce typologies. To ensure reliability, two researchers analysed the data independently and the results were compared. Both generated similar systems of categorisation, although these were initially described in slightly different terms. 


\section{Results}

All 24 respondents were general practitioner principals, and 10 were women. Their mean age was 43 (range 29-61). The eight practices had between three and eight partners, all but one were fundholding, and two were training practices.

\section{Definitions}

Responses to the question on how general practitioners defined effective health care fell into three broad categories: clinical, patient related, and resource related. Just over half the respondents (13) offered more than one type of definition.

\section{Clinical definitions}

Most of the respondents (18) offered definitions that were centred around appropriate investigation, treatment, referral, and follow up; improving morbidity and mortality; and curing and preventing disease. The definitions in this category included two distinct groups: firstly, definitions that were strictly focused on diseasesuch as, "We ought to be able to see that the disease process is treated"-and, secondly, definitions that incorporated a sense of the patient-such as, "That the patient has got better from whatever it was or that you've alleviated the suffering in some way."

Patient related definitions

Just under half the respondents (10) offered definitions that were more patient oriented. The most common theme was educating patients and giving them relevant information so that they were able to participate in the decision making process. For example, effectiveness was defined as "helping [patients] to come to a level of understanding such that they can personally make the decision about what happens to them." Three respondents referred to patient satisfaction as a condition of effectiveness. Another theme, offered by one respondent, was temporarily acquiescing to patients' expectations in order to secure compliance: "You might give somebody a big dose of something to make them better quickly so that they then believe you later when you want to change things."

\section{Resource related definitions}

Eight respondents gave definitions related to resources. These included ideas about cost effective care for individual patients and about a population perspective-for example: "All our decision making before used to be [that] a patient would come to you, and you just made a decision on the basis of that patient, but now I think there is a need for it to be made in a wider context."

\section{Reasons for not practising effectively}

Only one respondent thought he always practised effectively. The rest admitted departing from their own models of effective health care in everyday practice. Four categories of reasons for this emerged, with 15 respondents citing more than one category.

\section{Doctor related reasons}

Fourteen respondents mentioned factors that originated from the doctor, either as a professional or as an individual. Nine respondents cited self perceived shortcomings in knowledge, experience, and skills and how well these were applied in practice-for example: "I don't pretend to be up to date all the time" and "Pressure from conflicting ideas so that you don't really know if you are right or wrong." In addition, feelings of being tired, stressed, or unmotivated (what one doctor called "human failings") were referred to by five respondents-" [In] periods when I've been under enormous personal stress ... my referral patterns shoot up."

\section{Patient related reasons}

Seven respondents mentioned factors that emanated from patients. It was suggested that when patients presented with more than one problem, sometimes acknowledged and sometimes hidden, it was necessary to prioritise even if this meant ignoring some of the problems. One example given was that of an overweight smoker who had had a heart attack; the general practitioner thought that the smoking should be tackled first and the obesity left for later consideration. Alternatively, the effective treatment of one condition might exacerbate the symptoms of another. The example was given of a patient with severe coronary artery disease, gastrointestinal disease, and severe osteoarthritis, for whom appropriate treatment of the osteoarthritis with non-steroidal anti-inflammatory drugs might exacerbate the gastrointestinal problems. Two respondents also mentioned patients' noncompliance as an obstacle to practising effectively.

\section{Doctor and patient related reasons}

Eighteen respondents referred to factors associated with the interaction between doctor and patient. There was a pervading feeling that patients' cultural backgrounds, beliefs and attitudes, and levels of understanding resulted in certain expectations that sometimes clashed with the requirements of clinical effectiveness. A commonly cited example was patients demanding to be prescribed antibiotics for respiratory tract infections that were likely to be viral. Other examples were requesting investigations when the results were likely to be negative and requesting inappropriate referrals.

Various reasons were given for bowing to such demands. Firstly, respondents wanted to avoid conflict with their patients-"You might agree to investigate someone because you just can't stand this person nagging you on and on about their complaints." Secondly, they were keen to keep the "custom" of their patients. As one respondent observed, "Some doctors frighten their patients away because they're so blooming effective. So nobody goes round to see them, and they think they've got everything beautifully organised." Thirdly, respondents thought that they should respect patients' views and that patients could sometimes be "right" even if their views were not corroborated by scientific evidence. Fourthly, the placebo effect was mentioned as a reason for providing "ineffective" treatments, because it "helped the healing process." Finally, feelings of sympathy for patients led respondents to provide treatments of doubtful effectiveness-"Say it was my child, if he was suffering as much as that child is suffering, then I would certainly say, 'Well, look, I would much rather give him the benefit of having an 
antibiotic,' which is not the right thing to do, but I would do it."

In addition, problems were identified in providing effective care for patients who were felt to be "difficult." These were people who, for one reason or another, "defied" diagnosis and treatment-"You try every single approach and nothing has worked .... I think it's partly the personality of the patient." It was also acknowledged that personal prejudices could result in the doctor "ignoring" certain patients or devoting less time and effort to them than was necessary.

Environmental reasons

Factors extraneous to both doctor and patient were mentioned by 19 respondents. The concern most commonly referred to (by 13 respondents) was that of time, and the strength of feeling about this was considerable. Time was seen as hindering effectiveness across all its dimensions-"Time influences everything. It influences getting a history correctly, engaging with the patient if you don't know them well, building up some sort of rapport, discussing treatment options, examining them properly." Lack of time was felt by many respondents to result in ineffective practice because it led them to bow to inappropriate patient demands-"It may be a Friday afternoon, I want to rush off. I want to prevent this patient calling us back on Saturday afternoon, and I would prescribe antibiotics."

Lack of resources was the other main issue, mentioned by six respondents. This was thought to adversely affect various aspects of care, such as the doctor:patient ratio, the repeat prescribing system, district nursing services, hospital referrals, and operations. One respondent pointed out that a patient waiting for more than a year for a cataract operation may experience a fall and a fracture, but "we don't say, 'This is ascribed to the cataract or the delay in waiting lists."

\section{Questioning behaviour}

We asked the general practitioners to indicate the sources of information they use when they have unanswered clinical questions about particular patients. All 24 respondents mentioned a practice partner or a hospital doctor, or both. Recourse to literature (books, journals, use of a library) was mentioned by 10 respondents, referral to outpatient clinics by four, the internet by two, and the Medline database by one doctor only.

\section{Changes in clinical practice}

We also asked what changes individual respondents had made to their own clinical practice over the past few years. A total of 17 respondents recalled 39 changes, most recalling up to three changes. These ranged from switching to a different drug or using an investigative test for the first time to changing management (such as using a lower treatment threshold). Three main categories of reasons accounted for 25 of the changes, either alone or in combination. Contact with a hospital doctor or observation of hospital practice through seeing patients after their hospital visit was given as the sole reason for six changes, journal articles were cited as the sole reason for five changes, and scientific meetings were given as the reason for four. Four changes were attributed to literature combined with hospital contact, four to scientific meet- ings and hospital contact and two to literature and scientific meetings. Reference to journal articles was commonly along the lines of, "I remember reading something about it," and did not indicate a literature search or a critical appraisal process. Various reasons were given for the remainder of the changes. Five respondents spoke of a "crystallisation" or an "evolving process" incorporating several sources when elaborating on how the changes had come about.

\section{Discussion}

The findings of our study suggest that the central assumptions of the evidence based medicine paradigm may not be shared by many general practitioners, making its application in general practice problematic

\section{Limitations of study}

The 24 general practitioners who participated in our study formed a small sample that was neither random nor representative and came from only one NHS region. However, given the basis on which they joined the study, it seems reasonable to assume that their interest in clinical effectiveness would, if anything, be greater than that of most general practitioners.

\section{Doctors' concerns}

The respondents seemed to be acutely aware of, and sensitive to, patients' expectations and were inclined to judge their practice in terms not only of clinical outcome but also of a patient centred interpretation of quality. Thus, in situations where the requirements of clinical effectiveness openly clash with the preferences or circumstances of individual patients, the latter might take precedence in shaping general practitioners' actions. This concurs with theories on the "holistic" nature of general practice, in which biomedical, personal, and contextual perspectives converge in the decision making process. ${ }^{8}$ The linear decision making suggested by the model of evidence based medicine, informed chiefly by normative standards of clinical effectiveness, sits uneasily within this framework.

In general practice the doctor-patient encounter is a dynamic phenomenon underpinned by negotiation that takes account of the preoccupations of both parties. The fact that the doctor sometimes chooses to place more weight on the patient's agenda than on clinical evidence seems to be a rational strategy aimed at maintaining an important relationship. The maintenance of this relationship-which is likely to impact on the "healing" process ${ }^{9}$ - may be more important to general practitioners than staying within the bounds of a statistically defined consensus on clinical effectiveness.

\section{Doctors' information seeking}

When faced with clinical uncertainty the respondents in this study seemed to make more use of their colleagues or hospital doctors than of scientific literature. This finding is supported by those of Barrie and Ward, who found that "desktop" and human sources were used to answer most of the questions that general practitioners generated during consultations and that literature was little used. ${ }^{10}$

Contact with hospital colleagues and observation of their practice seems to have been as influential as lit- 
erature in prompting the respondents to change their clinical practice. Allery et al also found that most changes in practice reported by the clinicians in their study (which included consultants and general practitioners) had no educational basis, and literature was mentioned as a reason for change in less than 10\% of instances of change. ${ }^{11}$

Limitations of practising evidence based medicine There is a growing literature on the shortcomings of the evidence based medicine model in general practice, including the scope and nature of the evidence available and its limited applicability in this aspect of patient care. ${ }^{12-14}$ The difficulties in disseminating evidence, identifying the best format for it, and overcoming organisational barriers to implementing it have also been examined. ${ }^{15}$ Proponents of evidence based medicine have identified a number of problems and suggested ways of addressing these. ${ }^{5}$

Our findings are based on a limited investigation that formed a small part of a study primarily undertaken for another purpose. Nevertheless, they suggest that the applicability of the evidence based medicine approach in general practice may be limited for more fundamental reasons associated with the assumptions that the model makes about doctors' ways of thinking and behaviour. Firstly, general practitioners may not share evidence based medicine's overarching concern with clinical effectiveness but instead see it as one consideration in a wider framework that also takes account of service oriented concerns such as patient satisfaction and time management. Secondly, even when the concept of clinical effectiveness does come to the fore and leads to self evaluation and the identification of gaps in knowledge, practitioners may prefer to turn to human rather than written sources. Thirdly, even if more evidence based sources are sought and information obtained, it is unlikely to be applied to practice if it proves unacceptable to patients or incompatible with their other needs. Some of these factors have also emerged in other studies. ${ }^{16}$ Furthermore, "diagnosis by prognosis" and "diagnosis by therapeutic response", both common in the uncertain environment of general practice, ${ }^{17}$ may preclude the formulation of clear clinical questions demanded by the evidence based medicine model.

The suggested routes to practising evidence based medicine in a clinical setting-acquiring and using critical appraisal skills in everyday patient encounters or, to save time, using evidence based databases and guidelines ${ }^{18}$-fail to adequately comprehend the complex nature of general practice. There is doubtless a need to improve clinical quality in general practice, as in hospital medicine. But policies aimed at this objective need to take account of the concerns of practitioners and should be compatible with the nature of their work; furthermore, they need to be built on an empirical understanding of how knowledge comes to underpin practice, which may, for good reason, be far from any rationalist ideal.

We thank other members of the research group, especially Professor A Haines, Dr I Nazareth, and S Lister for their comments on the paper. The study was carried out in collaboration with the MRC General Practice Research Framework and we are grateful to all the general practitioners who participated.
Key messages

- Evidence based medicine has emerged as a new paradigm to prevent inappropriate variations in clinical practice

- This study explored the extent to which evidence based medicine's emphasis on clinical effectiveness, self analysis, and information seeking is congruent with the modes of thinking and behaviour of general practitioners

- General practitioners' definitions of effective health care fell into three categories of clinical, patient related, and resource related; their main reason for not practising effectively was patient factors, and others were lack of time, lack of knowledge and skills, lack of resources, and "human failings"; and their main sources of information in cases of clinical uncertainty were general practitioner partners and hospital doctors

- The central assumptions of the evidence based medicine paradigm may not be shared by many general practitioners, making its application in general practice problematic

- Promotion of effective care in general practice requires a broader vision and a more pragmatic approach that takes account of practitioners' concerns and is compatible with the complex nature of their work

Contributors: A Haines had the original idea for the feasibility study, initiated the research, and discussed core aspects of its design and execution. SR was overall coordinator of both design and execution of the feasibility study, organised recruitment of the study practices, and developed packs of evidence based guidelines used in the study. ZT and CH designed the qualitative component of the study, including the interviews reported on here. I Nazareth and S Lister developed and implemented the interventions strategies used in the feasibility study. ZT, CH, and SR participated in writing this paper. A Haines, I Nazareth, and $\mathrm{S}$ Lister commented on drafts of the paper. $\mathrm{CH}$ is guarantor for the paper.

Funding: The study was supported by a grant from the NHS Implementation Methods Programme.

Competing interest: None declared.

1 D'Annuo T, Vaughan TE. Variations in methadone treatment practices. Results from a national study. JAMA 1992;267:253-8.

2 Coulter A, Klassen A, MacKenzie IZ, McPherson K. Diagnostic dilatation and curettage: is it used appropriately? BMJ 1993;306:236-9.

3 Dunning $M$, Lugon M, MacDonald J. Is clinical effectiveness a management issue? BMJ 1998;316:243-4.

4 Milner P. A new national classification of health services based on clinical effectiveness. J Public Health Med 1997;19:127-8.

5 Evidence-Based Medicine Working Group. Evidence based medicine. JAMA 1992;268:2420-5.

6 Sackett DL, Rosenberg WMC, Gray JAM, Haynes RB, Richardson WS Evidence based medicine: what it is and what it isn't. BMJ 1996;312:71-2

7 Medical Research Council General Practice Research Framework. A network of general practices throughout the UK managed by the MRC Epidemiology and Medical Care Unit. London: EMCU, Wolfson Institute of Preventive Medicine, 1997.

8 Jacobson LD, Edwards AGK, Granier SK, Butler CC. Evidence-based medicine and general practice. Br J Gen Pract 1997;47:449-52.

9 Sullivan FM, MacNaughton RJ. Evidence in consultations: interpreted and individualised. Lancet 1996;348:941-3.

10 Barrie AR, Ward AM. Questioning behaviour in general practice: a pragmatic study. BMJ 1997;315:1512-5.

11 Allery LA, Owen PA, Robling MR. Why general practitioners and consultants change their clinical practice: a critical incident study. $B M J$ 1997;314:870-4.

12 Naylor CD. Grey zones of clinical practice: some limits to evidence-based medicine. Lancet 1995;345:840-2.

13 Kernick DP. Which antidepressant? A commentary from general practice on evidence-based medicine and health economics. Br J Gen Pract 1997;47:95-8.

14 Owen P. Clinical practice and medical research: bridging the divide between two cultures. Br J Gen Pract 1995;45:557-60.

15 Haines A. The science of perpetual change. BrJ Gen Pract 1996;46:115-9.

16 McColl A, Smith H, White P, Field J. General practitioners' perceptions of the route to evidence based medicine: a questionnaire survey. $B M J$ the route to evide
1998:316:361-5.

17 Greenhalgh T. "Is my practice evidence-based?" BMJ 1996;313:957-8.

18 Sackett DL, Rosenberg WMC. On the need for evidence-based medicine. J Public Health Med 1995;17:330-4.

(Accepted 29 January 1999) 